University of Nebraska - Lincoln

DigitalCommons@University of Nebraska - Lincoln

2012

\title{
The formulation and computation of the nonlocal J-integral in bond-based peridynamics
}

\author{
Wenke Hu \\ University of Nebraska-Lincoln \\ Youn Doh $\mathrm{Ha}$ \\ Kunsan National University, Korea \\ Florin Bobaru \\ University of Nebraska-Lincoln, fbobaru2@unl.edu \\ Stewart A. Silling \\ Sandia National Laboratories, NM
}

Follow this and additional works at: https://digitalcommons.unl.edu/mechengfacpub

Hu, Wenke; Ha, Youn Doh; Bobaru, Florin; and Silling, Stewart A., "The formulation and computation of the nonlocal J-integral in bond-based peridynamics" (2012). Mechanical \& Materials Engineering Faculty Publications. 80.

https://digitalcommons.unl.edu/mechengfacpub/80

This Article is brought to you for free and open access by the Mechanical \& Materials Engineering, Department of at DigitalCommons@University of Nebraska - Lincoln. It has been accepted for inclusion in Mechanical \& Materials Engineering Faculty Publications by an authorized administrator of DigitalCommons@University of Nebraska Lincoln. 


\title{
The formulation and computation of the nonlocal J-integral in bond-based peridynamics
}

\author{
Wenke Hu • Youn Doh Ha • Florin Bobaru • \\ Stewart A. Silling
}

Received: 31 January 2012 / Accepted: 14 June 2012 / Published online: 11 July 2012

(C) Springer Science+Business Media B.V. 2012

\begin{abstract}
This work presents a rigorous derivation for the formulation of the J-integral in bond-based peridynamics using the crack infinitesimal virtual extension approach. We give a detailed description of an algorithm for computing this nonlocal version of the J-integral. We present convergence studies ( $m$-convergence and $\delta$-convergence) for two different geometries: a single edge-notch configuration and a double edge-notch sample. We compare the results with results based on the classical J-integral and obtained from FEM calculations that employ special elements near the crack tip. We identify the size of the nonlocal region for which the peridynamic J-integral value is near the classical FEM solutions. We discuss how the boundary conditions and the peridynamic "skin effect" may influence the peridynamic J-integral value. We also observe, computationally, the path-independence of the peridynamic J-integral.
\end{abstract}

W. Hu · Y. D. Ha · F. Bobaru (凶)

Department of Mechanical \& Materials Engineering, University of Nebraska-Lincoln, Lincoln, NE 68588, USA e-mail: fbobaru2@unl.edu

\section{S. A. Silling}

Sandia National Laboratories, Multiphysics Simulation Technology Department, Albuquerque, NM 87185, USA

Present Address:

Y. D. Ha

Department of Naval Architecture, Kunsan National University, Kunsan, Korea
Keywords J-integral $\cdot$ Peridynamics $\cdot$ Nonlocal methods $\cdot$ Path-independence $\cdot$ Fracture

\section{Introduction}

The J-integral formulation for a linear elastic body in 2D was introduced by Rice (1968). The J-integral value was shown to be path independent and equivalent with the energy release rate. The J-integral can be understood both as a fracture energy parameter and as a stress intensity parameter because $\mathrm{J}$ uniquely characterizes crack-tip stresses and strains (Hutchinson 1968; Rice and Rosengren 1968). The J-integral has been extensively used to compute energy flow to the crack tip, to estimate crack opening and as part of failure criteria for ductile materials. Recently, the peridynamic theory was introduced in order to handle problems with discontinuous fields (Silling 2000). Peridynamics is a nonlocal formulation of classical mechanics which allows for a natural treatment of crack initiation, growth and propagation. The peridynamic theory has been successfully applied to damage analysis of viscoplastic materials (Foster et al. 2010, 2011) dynamic fracture and crack branching in glass (Ha and Bobaru 2010, 2011), damage in composite materials from impact or shock loading (Xu et al. 2008; Kilic et al. 2009; Hu et al. 2011, 2012) and nano-scale structures (Silling and Bobaru 2005; Bobaru 2007). The main motivation for studying the J-integral in peridynamics is that it allows computation of the energy consumed by a growing crack for any bond failure criterion, and for any dissipative 
mechanisms and nonlinearities that may be occurring, provided these occur sufficiently close to the crack tip. Also, the J-integral itself could be used as a failure criterion in computations: it is possible to use a critical value of $\mathrm{J}$ as a criterion for breaking bonds.

While for problems in elastic domains without cracks, peridynamics converges to classical elasticity in the limit of the nonlocal region (the horizon) going to zero, it is important to determine the size of this region for which the peridynamic J-integral results are close to those obtained from the classical J-integral. Silling and Lehoucq (2010) presented a state-based peridynamic J-integral formulation based on energy balance approach and related it to the Griffith criterion. In the present study, we derive the nonlocal J-integral for the bond-based peridynamic formulation by means of an infinitesimal virtual crack extension. We describe an algorithm for computing this nonlocal version of the J-integral and perform several convergence studies in order to find the size of the nonlocal region (the peridynamic horizon size) for which the nonlocal Jintegral value is nearing the classical J-integral value. We also investigate the dependence of the peridynamic J-integral value on: (1) the boundary conditions; (2) the peridynamic "skin effect", and (3) the location of the integral contour (a path-independence study). We compare our results with results obtained from FEM solutions of the classical Linear Fracture Mechanics using special elements. The special elements use the exact analytical solution of linear elastic fracture mechanics.

The paper is organized as follows: in Sect. 2, we review the basic formulation for bond-based peridynamics; in Sect. 3 we give the derivation for the peridynamic J-integral based on the infinitesimal virtual crack extension; in Sect. 4 we present the algorithm used for computing the peridynamic J-integral; in Sect. 5, we perform two types of convergence ( $m$-convergence and $\delta$-convergence) studies for a thin plate with single edge notch and double edge notch and we compare the peridynamic results with those obtained from finite element calculations for the classical J-integral using Abaqus 6.10. Calculations related to the path-independence of the nonlocal J-integral are also shown. Conclusions are given in Sect. 6.

\section{Introduction to the peridynamic theory}

The peridynamic equations of motion are given as $\rho \ddot{\mathbf{u}}(\mathbf{x}, t)=\int_{H} \mathbf{f}(\mathbf{u}(\hat{\mathbf{x}}, t)-\mathbf{u}(\mathbf{x}, t), \hat{\mathbf{x}}-\mathbf{x}) d V_{\hat{\mathbf{x}}}+\mathbf{b}(\mathbf{x}, t)$

where $\mathbf{f}$ is the pairwise force function in the peridynamic bond that connects point $\hat{\mathbf{x}}$ to $\mathbf{x}$, and $\mathbf{u}$ is the displacement vector field. $\rho$ is the density and $\mathbf{b}$ is the body force intensity. The integral is defined over a region $H$ called the "horizon region". The horizon region is the compact supported domain of the pairwise force function around a point $\mathbf{x}$, and is taken most often as a disk in 2D applications (and sphere in 3D). We will abuse the language and call the radius $\delta$ of such a disk the horizon. Then, the meaning of the word "horizon" should be clear from the context. The interpretation, selection, and use of the peridynamic horizon and its relation to dynamic crack propagation is discussed in Bobaru and $\mathrm{Hu}$ (2012).

A micro-elastic material (Silling 2000) is defined as one for which the pairwise force derives from a potential $\omega$ :

$\mathbf{f}(\boldsymbol{\eta}, \boldsymbol{\xi})=\frac{\partial \omega(\boldsymbol{\eta}, \boldsymbol{\xi})}{\partial \boldsymbol{\eta}}$

where $\boldsymbol{\xi}=\hat{\mathbf{x}}-\mathbf{x}$ is the relative position in the reference configuration and $\boldsymbol{\eta}=\hat{\mathbf{u}}-\mathbf{u}$ is the relative displacement.

A linear micro-elastic potential, which leads to a linear relationship between the bond force and the relative elongation of the bond, is obtained if we take

$\omega(\boldsymbol{\eta}, \boldsymbol{\xi})=\frac{c(\|\boldsymbol{\xi}\|) s^{2}\|\boldsymbol{\xi}\|}{2}$

where $s$ the bond relative elongation

$s=\frac{\|\xi+\eta\|-\|\xi\|}{\|\xi\|}$

The corresponding pairwise force becomes

$\mathbf{f}(\boldsymbol{\eta}, \boldsymbol{\xi})=\frac{\partial \omega(\boldsymbol{\eta}, \boldsymbol{\xi})}{\partial \boldsymbol{\eta}}=c(\|\boldsymbol{\xi}\|) s \frac{\partial\|\boldsymbol{\xi}+\boldsymbol{\eta}\|}{\partial \boldsymbol{\eta}}$

with

$\frac{\partial\|\boldsymbol{\xi}+\eta\|}{\partial \eta}=\mathbf{e}$

where $\mathbf{e}$ is the unit vector along the direction of the deformed bond, $\boldsymbol{\xi}+\boldsymbol{\eta}$, between $\hat{\mathbf{x}}$ and $\mathbf{x}$ in the deformed configuration.

The function $c(\|\xi\|)$ is called micromodulus function and it represents the bond elastic stiffness. Some possible choices for the micromodulus function are 


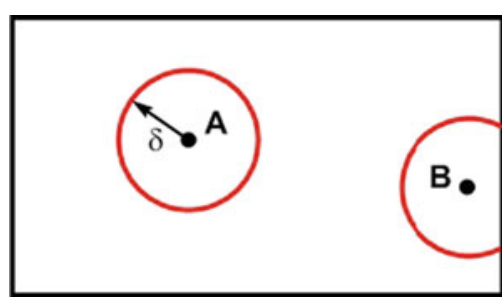

Fig. 1 The skin effect in peridynamics for points close to the boundary

given in 1D (Bobaru et al. 2009), 2D (Ha and Bobaru 2010) and 3D (Silling and Askari 2005). These are obtained by matching [see Eq. (6)] the elastic strain energy density at a material point in the bulk (see point $\mathbf{A}$ in Fig. 1) of a microelastic peridynamic material to the elastic strain energy density from the classical theory, when both materials are under the same homogeneous deformation.

$W_{\text {classical }}=\frac{1}{2} \int_{H} \frac{c(\|\boldsymbol{\xi}\|) s^{2}\|\boldsymbol{\xi}\|}{2} d A_{\hat{\mathbf{x}}}$

For simplicity, we use the form of the micromodulus function obtained for a point in the bulk for all points, including those that are within $\delta$ from the surface (like point B in Fig. 1). This leads to an effectively softer material close to the boundary, since now the integral in Eq. (6) is over a smaller region and for the match to hold one would have to increase the micromodulus value. Therefore, the strains will be larger for the "skin" of the domain than in the bulk of the material, for a deformation that classically would be homogeneous (see Bobaru and $\mathrm{Ha}$ 2011). We call this behavior in peridynamics the "skin effect". The skin effect can be compensated for by applying a correction factor to the micromodulus of bonds near a surface (Macek and Silling 2007; Kilic 2008). A similar observation can be made about the effect of surfaces on the critical relative elongation parameter that is correlated with the fracture energy of a material, as discussed in Ha and Bobaru (2011).

In this paper, we use the "conical" micromodulus functions given below (see Fig. 2) since it gives better convergence rates to the classical (local) solutions in elasticity problems compared to the simpler, constant micromodulus (see Bobaru et al. 2009; Ha and Bobaru 2010).

$c(\|\boldsymbol{\xi}\|)=C_{1}\left(1-\frac{\|\boldsymbol{\xi}\|}{\delta}\right)=\frac{24 E}{\pi \delta^{3}(1-v)}\left(1-\frac{\|\boldsymbol{\xi}\|}{\delta}\right)$

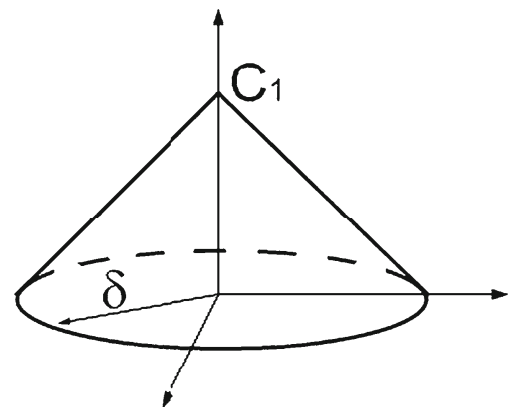

Fig. 2 The conical micromodulus function

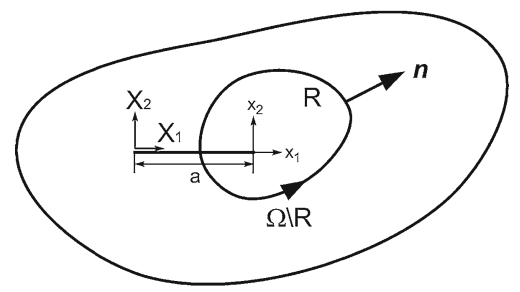

Fig. 3 Two-dimensional body containing a straight crack

where $E$ and $v$ are Young's modulus and Poisson's ratio, respectively. $\delta$ is horizon size.

\section{Derivation of the peridynamic J-integral}

In this section we derive the peridynamic J-integral for a mode I crack that grows in a self similar manner in a 2D microelastic peridynamic body. We consider a certain domain $\Omega$, which contains a straight crack of length $a$, in equilibrium and zero body force density. The peridynamic equations are:

$\int_{\Omega} \mathbf{f}(\mathbf{u}(\hat{\mathbf{x}}, t)-\mathbf{u}(\mathbf{x}, t), \hat{\mathbf{x}}-\mathbf{x}) d A_{\hat{\mathbf{x}}}=0$

where $A_{\hat{\mathbf{x}}}$ represents the nodal area in 2D instead nodal volume $V_{\hat{\mathbf{x}}}$ in $3 \mathrm{D}$.

The elastic energy density at any $\mathrm{x}$ in $\Omega$ for the microelastic material is

$W(\mathbf{x} ; \mathrm{a})=\frac{1}{2} \int_{\Omega(\mathrm{a})} \omega(\boldsymbol{\eta}, \boldsymbol{\xi}) d A_{\hat{\mathbf{x}}}$

where $a$ is the crack length (see Fig. 3).

Consider a fixed coordinate system $\left(\mathrm{X}_{1}, \mathrm{X}_{2}\right)$ as in Fig. 3. The local coordinate system $\left(\mathrm{x}_{1}, \mathrm{x}_{2}\right)$ is attached to the crack tip and moves as the crack grows. Assume that the crack grows by an infinitesimal amount in the $\mathrm{x}_{1}$ 
direction. Considering the change in total elastic strain energy resulting from an infinitesimal virtual extension of the crack we get:

$\frac{d U}{d \mathrm{a}}=-\frac{d}{d \mathrm{a}} \oint_{R(\mathrm{a})} W(\mathbf{x} ; \mathrm{a}) d A_{\mathbf{x}}$

The right hand side of Eq. (10), by adding and subtracting $\oint_{R(\mathrm{a})} W(\mathbf{x} ; \mathrm{a}+\Delta \mathrm{a}) d A_{\mathbf{x}}$, can be written as:

$$
\begin{aligned}
& \frac{d}{d \mathrm{a}} \oint_{R(\mathrm{a})} W(\mathbf{x} ; \mathrm{a}+\Delta \mathrm{a}) d A_{\mathbf{x}}=\lim _{\Delta \mathrm{a} \rightarrow 0} \frac{1}{\Delta \mathrm{a}} \\
& \times \oint_{R(\mathrm{a}+\Delta \mathrm{a})-\mathrm{R}(\mathrm{a})} W(\mathbf{x} ; \mathrm{a}+\Delta \mathrm{a}) d A_{\mathbf{x}} \\
& \left.\quad+\oint_{R(\mathrm{a})} W(\mathbf{x} ; \mathrm{a}+\Delta \mathrm{a})-W(\mathbf{x} ; \mathrm{a}) d A_{\mathbf{x}}\right] \\
& =\oint_{\partial R} W(\mathbf{x} ; \mathrm{a}) \mathrm{n}_{1} d S+\oint_{R(\mathrm{a})} \frac{\partial W(\mathbf{x} ; \mathrm{a})}{\partial \mathrm{a}} d A_{\mathbf{x}}
\end{aligned}
$$

Consider now the second term on the right hand side of Eq. (11) and by using Eqs. (9) and (2), we have

$$
\begin{aligned}
\oint_{R(\mathrm{a})} & \frac{\partial W(\mathbf{x} ; \mathrm{a})}{\partial \mathrm{a}} d A_{\mathbf{x}} \\
= & \frac{1}{2} \oint_{R(\mathrm{a})} \oint_{\Omega(\mathrm{a})} \mathbf{f}(\boldsymbol{\eta}, \boldsymbol{\xi}) \cdot\left(\frac{\partial \hat{\mathbf{u}}}{\partial \mathrm{a}}-\frac{\partial \mathbf{u}}{\partial \mathrm{a}}\right) d A_{\mathbf{x}} \\
= & \frac{1}{2} \oint_{R(\mathrm{a})} \oint_{\Omega(\mathrm{a})} \mathbf{f}(\boldsymbol{\eta}, \boldsymbol{\xi}) \cdot \frac{\partial \hat{\mathbf{u}}}{\partial \mathrm{a}} d A_{\hat{\mathbf{x}}} d A_{\mathbf{x}} \\
& -\frac{1}{2} \oint_{R(\mathrm{a})} \oint_{\Omega(\mathrm{a})} \mathbf{f}(\boldsymbol{\eta}, \boldsymbol{\xi}) d A_{\hat{\mathbf{x}}} d A_{\hat{\mathbf{x}}} \frac{\partial \mathbf{u}}{\partial \mathrm{a}} d A_{\mathbf{x}}
\end{aligned}
$$

The second term on the right hand side of Eq. (12) is equal to zero because of Eq. (8). Thus, Eq. (12) reduces to

$$
\oint_{R(\mathrm{a})} \frac{\partial W(\mathbf{x} ; \mathrm{a})}{\partial \mathrm{a}} d A_{\mathbf{x}}=\frac{1}{2} \oint_{\Omega(\mathrm{a})} \oint_{R(\mathrm{a})} \mathbf{f}(\boldsymbol{\eta}, \boldsymbol{\xi}) d A_{\mathbf{x}} \frac{\partial \hat{\mathbf{u}}}{\partial \mathrm{a}} d A_{\hat{\mathbf{x}}}
$$

Defining the domain $\mathrm{Q}(\mathrm{a})=\Omega(\mathrm{a}) \backslash \mathrm{R}(\mathrm{a})$, the right hand side of Eq. (13) can be rewritten as

$$
\begin{aligned}
& \frac{1}{2} \oint_{\Omega(\mathrm{a})} \oint_{R(\mathrm{a})} \mathbf{f}(\boldsymbol{\eta}, \boldsymbol{\xi}) d A_{\mathbf{x}} \frac{\partial \hat{\mathbf{u}}}{\partial \mathrm{a}} d A_{\hat{\mathbf{x}}} \\
& =\frac{1}{2} \oint_{Q(\mathrm{a})} \oint_{R(\mathrm{a})} \mathbf{f}(\boldsymbol{\eta}, \boldsymbol{\xi}) d A_{\mathbf{x}} \frac{\partial \hat{\mathbf{u}}}{\partial \mathbf{a}} d A_{\hat{\mathbf{x}}} \\
& \quad+\frac{1}{2} \oint_{R(\mathrm{a})} \oint_{R(\mathrm{a})} \mathbf{f}(\boldsymbol{\eta}, \boldsymbol{\xi}) d A_{\mathbf{x}} \frac{\partial \hat{\mathbf{u}}}{\partial \mathrm{a}} d A_{\hat{\mathbf{x}}}
\end{aligned}
$$

Also, from Eq. (8), we have

$$
\oint_{R(\mathrm{a})} \mathbf{f}(\boldsymbol{\eta}, \boldsymbol{\xi}) d A_{\hat{\mathbf{x}}}=-\oint_{Q(\mathrm{a})} \mathbf{f}(\boldsymbol{\eta}, \boldsymbol{\xi}) d A_{\hat{\mathbf{x}}}
$$

By the change of variables $\hat{\mathbf{x}} \rightarrow \mathbf{x}$ and using the linear admissibility condition (see Silling 2000)

$\mathbf{f}(-\eta,-\xi)=-\mathbf{f}(\eta, \xi)$

and employing Eqs. (15), (14) can be written as

$$
\begin{aligned}
& \frac{1}{2} \oint_{\Omega(\mathrm{a})} \oint_{R(\mathrm{a})} \mathbf{f}(\boldsymbol{\eta}, \boldsymbol{\xi}) d A_{\mathbf{x}} \frac{\partial \hat{\mathbf{u}}}{\partial \mathrm{a}} d A_{\hat{\mathbf{x}}} \\
= & \frac{1}{2} \oint_{Q(\mathrm{a})} \oint_{R(\mathrm{a})} \mathbf{f}(\boldsymbol{\eta}, \boldsymbol{\xi}) d A_{\mathbf{x}} \frac{\partial \hat{\mathbf{u}}}{\partial \mathrm{a}} d A_{\hat{\mathbf{x}}} \\
& +\frac{1}{2} \oint_{R(\mathrm{a})} \oint_{R(\mathrm{a})} \mathbf{f}(-\boldsymbol{\eta},-\boldsymbol{\xi}) d A_{\hat{\mathbf{x}}} \frac{\partial \mathbf{u}}{\partial \mathrm{a}} d A_{\mathbf{x}} \\
= & \frac{1}{2} \oint_{Q(\mathrm{a})} \oint_{R(\mathrm{a})} \mathbf{f}(\boldsymbol{\eta}, \boldsymbol{\xi}) d A_{\mathbf{x}} \frac{\partial \hat{\mathbf{u}}}{\partial \mathrm{a}} d A_{\hat{\mathbf{x}}} \\
& -\frac{1}{2} \oint_{R(\mathrm{a})} \oint_{R(\mathrm{a})} \mathbf{f}(\boldsymbol{\eta}, \boldsymbol{\xi}) d A_{\hat{\mathbf{x}}} \frac{\partial \mathbf{u}}{\partial \mathrm{a}} d A_{\mathbf{x}} \\
= & \frac{1}{2} \oint_{R(\mathrm{a})} \oint_{Q(\mathrm{a})} \mathbf{f}(\boldsymbol{\eta}, \boldsymbol{\xi}) \cdot \frac{\partial \hat{\mathbf{u}}}{\partial \mathrm{a}} d A_{\hat{\mathbf{x}}} d A_{\mathbf{x}} \\
& +\frac{1}{2} \oint_{R(\mathrm{a})} \oint_{Q(\mathrm{a})} \mathbf{f}(\boldsymbol{\eta}, \boldsymbol{\xi}) \cdot \frac{\partial \mathbf{u}}{\partial \mathrm{a}} d A_{\hat{\mathbf{x}}} d A_{\mathbf{x}}
\end{aligned}
$$

Substituting Eq. (17) into Eq. (12), we get

$$
\begin{aligned}
& \oint_{R(\mathrm{a})} \frac{\partial W(\mathbf{x} ; \mathrm{a})}{\partial \mathrm{a}} d A_{x} \\
& =\frac{1}{2} \oint_{R(\mathrm{a})} \oint_{\Omega(\mathrm{a}) \backslash \mathrm{R}(\mathrm{a})} \mathbf{f}(\boldsymbol{\eta}, \boldsymbol{\xi}) \cdot\left(\frac{\partial \hat{\mathbf{u}}}{\partial \mathrm{a}}+\frac{\partial \mathbf{u}}{\partial \mathrm{a}}\right) d A_{\hat{\mathbf{x}}} d A_{\mathbf{x}}
\end{aligned}
$$




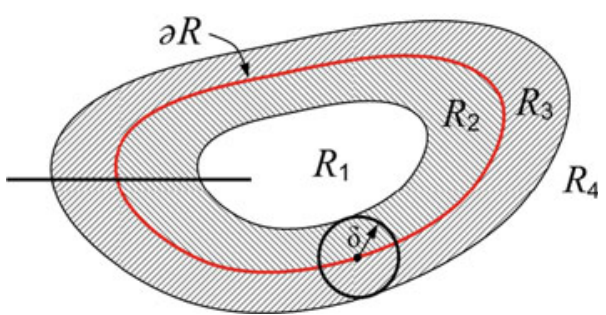

Fig. 4 Integration domain for the peridynamic J-integral. The red curve is the contour of integration

Since the coordinates $\left(\mathrm{x}_{1}, \mathrm{x}_{2}\right)$ are attached at the crack tip, and are defined by

$\mathrm{x}_{1}=\mathrm{X}_{1}-\mathrm{a}, \quad \mathrm{x}_{2}=\mathrm{X}_{2}$

we have that $\frac{\partial \mathrm{x}_{1}}{\partial \mathrm{a}}=-1$

By using the chain rule, we obtain

$\frac{\partial \hat{\mathbf{u}}}{\partial \mathrm{a}}=-\frac{\partial \hat{\mathbf{u}}}{\partial \mathrm{x}_{1}}, \quad \frac{\partial \mathbf{u}}{\partial \mathrm{a}}=-\frac{\partial \mathbf{u}}{\partial \mathrm{x}_{1}}$

Then, we place Eq. (19), (18), and (11) back into Eq. (10) to get the peridynamic J-integral formula as follow:

$$
\begin{aligned}
J_{\text {peri }}= & \oint_{\partial R} W(\mathbf{x} ; \mathrm{a}) \mathrm{n}_{1} d S-\frac{1}{2} \oint_{R(\mathrm{a})} \oint_{\Omega(\mathrm{a}) \backslash \mathrm{R}(\mathrm{a})} \mathbf{f}(\boldsymbol{\eta}, \boldsymbol{\xi}) \\
& \cdot\left(\frac{\partial \hat{\mathbf{u}}}{\partial \mathrm{x}_{1}}+\frac{\partial \mathbf{u}}{\partial \mathrm{x}_{1}}\right) d A_{\hat{\mathbf{x}}} d A_{\mathbf{x}}
\end{aligned}
$$

where $W(\mathbf{x} ; a)$ is the strain energy density $\partial R$ is the integral contour. $R(a)$ is the region inside the integral contour, and $\Omega(\mathrm{a}) \backslash R(a)$ is the region outside the integral contour (see Fig. 3). Silling and Lehoucq (2010) obtained the state-based peridynamic J-integral formulation based on an energy approach. We observe that in the particular case of the bond-based theory, the formula in Silling and Lehoucq (2010) coincides with the one we obtained here in Eq. (20).

Discussion: The first term of peridynamic J-integral [see Eq. (20)] is a contour integral, along $\partial R$, where $R=R_{1} \mathrm{U} R_{2}$ (see red curve in Fig. 4). The set $R_{2}$ is a "band" of thickness $\delta$ inside the contour $\partial R$, and $R_{3}$ is a band of thickness $\delta$ outside of the contour $\partial R$. The second term of the peridynamic J-integral is a double domain integral. This domain integral is zero unless the points in $R$ are in $R_{2}$, and the points in $\Omega \backslash R$ are in $R_{3}$. Thus, Eq. (20) can be written as follows

$$
\begin{aligned}
J_{\text {peri }}= & \oint_{\partial R} W(\mathbf{x} ; \mathrm{a}) \mathrm{n}_{1} d S \\
& -\frac{1}{2} \oint_{R_{2}} \oint_{R_{3}} \mathbf{f}(\boldsymbol{\eta}, \boldsymbol{\xi}) \cdot\left(\frac{\partial \hat{\mathbf{u}}}{\partial \mathrm{x}_{1}}+\frac{\partial \mathbf{u}}{\partial \mathrm{x}_{1}}\right) d A_{\hat{\mathbf{x}}} d A_{\mathbf{x}}
\end{aligned}
$$

Clearly, when the horizon $\delta$ goes to zero, the domain integral becomes a contour integral and the regions $R_{2}$ and $R_{3}$ reduce to the contour $\partial R$. In this case, the formulation of peridynamic $J$ integral coincides with the classical J-integral formula. Indeed, the peridynamic J-integral formulation when horizon goes to zero is

$J_{\text {peri }}=\oint_{\partial R} W(\mathbf{x} ; \mathrm{a}) \mathrm{n}_{1} d S-\oint_{\partial R} \frac{\partial \mathbf{u}}{\partial \mathrm{x}_{1}} \cdot \boldsymbol{\tau}(\mathbf{x}, \mathbf{n}) d S$

where $\boldsymbol{\tau}(\mathbf{x}, \mathbf{n})$ is the force flux vector at any $\mathbf{x}$ in the direction of unit vector $\mathbf{n}$ normal to the tangent of the contour (see Lehoucq and Silling 2008).

Remark Notice that for a fixed horizon size, the contour needs to be large enough so that the regions $R_{2}$ and $R_{3}$ exist as shown in Fig. 4. Examples of feasible and unfeasible contours are shown in Sect. 5.

\section{An algorithm for calculating the peridynamic J-integral}

We use a uniform discretization with grid spacing $\Delta x$ over the domain to compute the peridynamic J-integral. Non-uniform discretizations (and even variable horizon size) are possible in peridynamics, see Bobaru and Ha 2011, but for simplicity here we use uniform grids. Using the mid-point integration scheme for the domain integral in Eq. (20) and the trapezoidal rule for the contour integral in Eq. (20) for the case when the contour is piecewise linear, such as the rectangular box in Fig. 6, leads to the following approximation in 2D:

$$
\begin{aligned}
J_{\text {peri }} & \approx \sum_{n=1}^{n_{\text {boundary }}} W n_{1} \Delta x \\
- & \frac{1}{2} \sum_{i=1}^{n_{\text {inner }}} \sum_{j=1}^{n_{\text {outer }}}\left(f\left(u_{1}^{j}-u_{1}^{i}, x_{1}^{j}-x_{1}^{i}\right)\left(\frac{\partial u_{1}}{\partial x_{1}}+\frac{\partial \hat{u}_{1}}{\partial x_{1}}\right)\right. \\
& \left.+f\left(u_{2}^{j}-u_{2}^{i}, x_{2}^{j}-x_{2}^{i}\right)\left(\frac{\partial u_{2}}{\partial x_{1}}+\frac{\partial \hat{u}_{2}}{\partial x_{1}}\right)\right) A_{j} A_{i}
\end{aligned}
$$

where $n_{\text {contour }}$ and $n_{\text {inner }}$ are the number of nodes along the integral contour boundary $\partial R$ and the number of nodes in the inner region (the $R_{2}$ region in Fig. 4), 


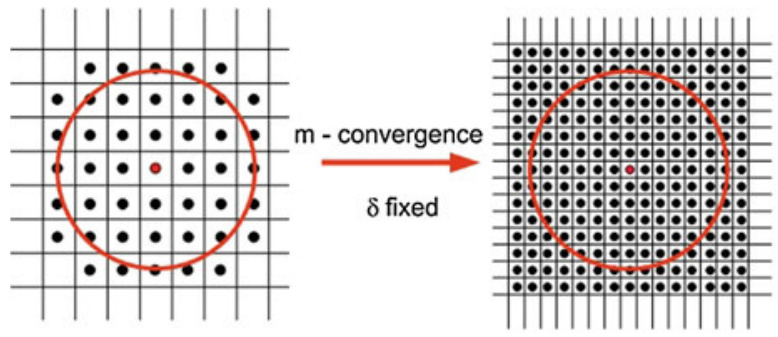

(a) $m$-convergence

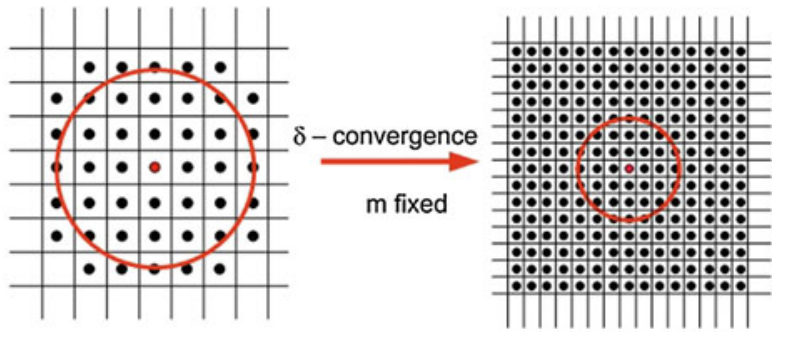

(b) $\delta$-convergence

Fig. 5 Graphical description for the $m$-convergence and $\delta$ - convergence in peridynamics. $m=\delta / \Delta \mathrm{x}$

respectively. $n_{\text {outer }}$ is the number of nodes in the outer region (the $R_{3}$ region in Fig. 4). $A_{i}$ is the nodal area or node $i$. The central difference scheme is used for $\partial \mathbf{u} / \partial \mathrm{x}_{1}$ and $\partial \hat{\mathbf{u}} / \partial \mathrm{x}_{1}$ as follows

$$
\begin{aligned}
& \frac{\partial u_{1}\left(x_{1}^{i}, x_{2}^{i}\right)}{\partial x_{1}^{i}} \approx \frac{u_{1}\left(x_{1}^{i}+\Delta x, x_{2}^{i}\right)-u_{1}\left(x_{1}^{i}-\Delta x, x_{2}^{i}\right)}{2 \Delta x}, \\
& \frac{\partial u_{2}\left(x_{1}^{i}, x_{2}^{i}\right)}{\partial x_{1}^{i}} \approx \frac{u_{2}\left(x_{1}^{i}+\Delta x, x_{2}^{i}\right)-u_{2}\left(x_{1}^{i}-\Delta x, x_{2}^{i}\right)}{2 \Delta x} \\
& \frac{\partial \hat{u}_{1}\left(x_{1}^{j}, x_{2}^{j}\right)}{\partial x_{1}^{j}} \approx \frac{\hat{u}_{1}\left(x_{1}^{j}+\Delta x, x_{2}^{j}\right)-\hat{u}_{1}\left(x_{1}^{j}-\Delta x, x_{2}^{j}\right)}{2 \Delta x}, \\
& \frac{\partial \hat{u}_{2}\left(x_{1}^{j}, x_{2}^{j}\right)}{\partial x_{1}^{j}} \approx \frac{\hat{u}_{2}\left(x_{1}^{j}+\Delta x, x_{2}^{j}\right)-\hat{u}_{2}\left(x_{1}^{j}-\Delta x, x_{2}^{j}\right)}{2 \Delta x}
\end{aligned}
$$

We compute the peridynamic J-integral with the following algorithm based on Eq. (21).

\section{Numerical examples: convergence studies, path-independence, and effects from the boundaries}

In peridynamics, two types of convergence studies are typically used: the $\delta$-convergence (fix the number of nodes covered by a horizon, which is proportional to $m=\delta / \Delta x$, and decrease the horizon size), and $m$-convergence (keep the horizon size fixed and increase $m$ ) (See Fig. 5 and Bobaru et al. 2009). It is important to note that in $m$-convergence studies, the peridynamic approximate solutions are not supposed to converge to the classical solution, but to the nonlocal solution for the particular horizon size for which the $m$-convergence study is executed. In this instance, comparing the results with the classical solution is only made for illustration purposes. Convergence of the peridynamic nonlocal solutions to the classical solution is expected in the limit of the horizon going to zero. In this paper we perform convergence studies to investigate for what horizon size, relative to the size of the sample, and which $m$-values does the peridynamic J-integral get close (with a relative difference of a few percentages) to the classical value of the J-integral. We then analyze the influence of boundary conditions (when symmetry conditions are used) and of the peridynamic "skin effect" (see Sect. 2) on the nonlocal J-integral. We compare the peridynamic results with the classical J-integral value as approximately given by Finite Element results using Abaqus with special crack-tip elements for a plate with an edge notch under tension. In order to investigate how symmetric boundary conditions influence the peridynamic J-integral value we analyze a double-edge notch plate under uniform tension, for which we use symmetry boundary conditions. We also perform calculations that show the path-independence of the peridynamic J-integral (Table 1).

In following examples, we use the same material parameters: Young's modulus is $72 \mathrm{GPa}$ and Poisson's ratio is $1 / 3$. Along top and bottom boundaries of the two different samples, a uniform tensile stress $\sigma=1 \mathrm{MPa}$ is applied. Uniform discretization is used for all computations.

In order to test the correctness of the implementation, we compute the peridynamic J-integral for a plate (see Fig. 6) without a notch, using the integral contour in Fig. 6. In this case, peridynamic J-integral values are in the range of $10^{-15}$ for any $m$-values and horizon size we tried. For instance, the peridynamic J-integral value is $3.3 \times 10^{-15} \mathrm{~Pa} \mathrm{~m}$ for $m=9$ and $\delta=1.5 \mathrm{~mm}$. The J-integral on a closed contour in an elastic material is zero. 
Table 1 Algorithm to compute the peridynamic J-integral

1: Get the nodal displacements and elastic strain energy density at all nodes

2: Define the geometry of the integral contour

3: Compute the first term (contour integral) of the peridynamic J-integral

(a) Find nodes along the contour $\partial R$ and the corresponding outer normal vector to the integral contour at such nodes

(b) Evaluate the line integral using trapezoidal rule

4: Compute the second term (domain double integral) of the peridynamic $\mathrm{J}$ integral

(a) Define the inner region $\left(R_{2}\right)$ : one horizon size inside of the integral contour $\partial R$

(b) Define the outer region $\left(R_{3}\right)$ : one horizon size outside of the integral contour $\partial R$

(c) Search all viable source nodes $\mathbf{x}^{\mathrm{i}}$ in inner region

(d) Search all viable nodes $\mathbf{x}^{\mathrm{j}}$ in outer region

(e) Compute the second term based on the discretization in Eq. (21)

5: $J_{\text {peri }}=$ First_term-Second_term

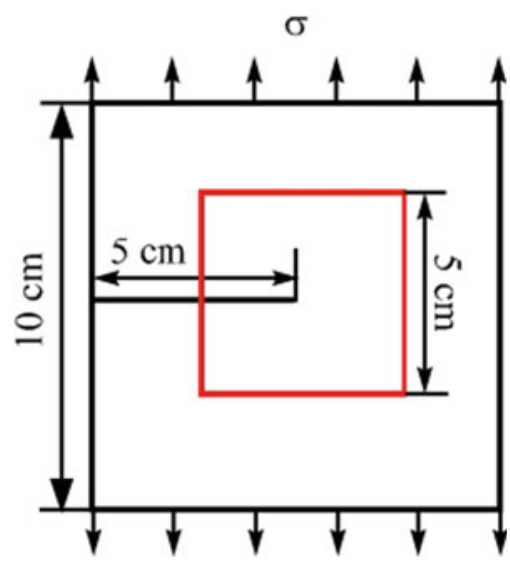

Fig. 6 Geometry configuration for single edge-notched plate (red square is the J-integral contour)

\subsection{Single-notched specimen}

Consider a single edge-notch square plate with dimension of $10 \mathrm{~cm} \times 10 \mathrm{~cm}$ with a length of the notch of $5 \mathrm{~cm}$, as shown in Fig. 6. We use the square contour shown in Fig. 6 to compute the peridynamic J-integral and the corresponding classical value with Abaqus. In Abaqus, we use the "seam crack" option to create the crack and use special crack-tip quadratic elements around the crack tip as shown in Fig. 7. In the actual FEM computation, the total number of nodes is about 30,000 (many more nodes than shown in Fig. 7), which

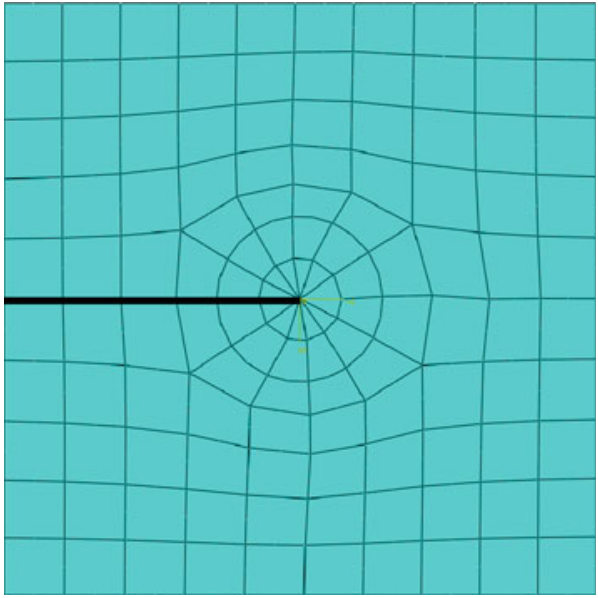

Fig. 7 A sample Abaqus mesh with special elements around the crack tip, used to compute the classical J-integral value. The dark solid line is the crack

gives us a converged value for the classical J-integral of about 19.76 Pam.

Since the J-integral involves the components of the displacement field, it is of interest to compare the displacements obtained from peridynamics with a "large" and a "small" horizon size with the classical displacements obtained from a sufficiently fine FEM mesh. As shown in Figs. 8 and 9, the x- and y-displacements from peridynamic computation are almost identical to the FEM calculations when the horizon size, relative to the sample size and crack size is below 1/20. In these figures the same legend is used for both the peridynamic results and the finite element results. The legend levels are produced automatically by Abaqus, and we set the same values in Tecplot to post-process the peridynamic results.

Two types of convergence studies ( $m$-convergence and $\delta$-convergence) are shown in Table 2 and Fig. 10. For a fixed $m$, the peridynamic J-integral approaches the classical solution when the horizon size decreases, so we do observe $\delta$-convergence to the classical J-integral value. We also note that the $m$-convergence curves (for a fixed $\delta$ ) start to level off when $m$ becomes larger than 6. Increasing $\mathrm{m}$ for a fixed horizon size delivers solutions that approach the exact nonlocal solution. The results of Fig. 10 indicate good candidates for both $\delta$ and $m$ to use in order to obtain the peridynamic J-integral values within a few percentages from the FEM results. We stress that, in general, the FEM results may also be a few percentages away from an analytical solution 
(a)

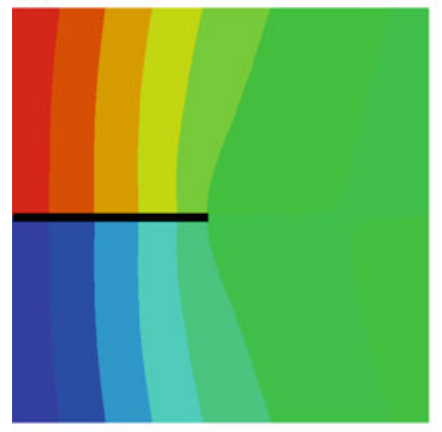

(b)

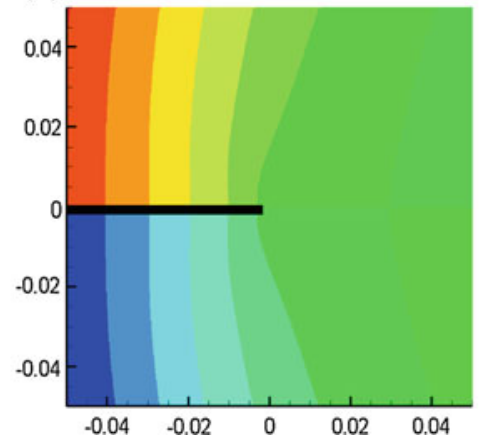

(c)

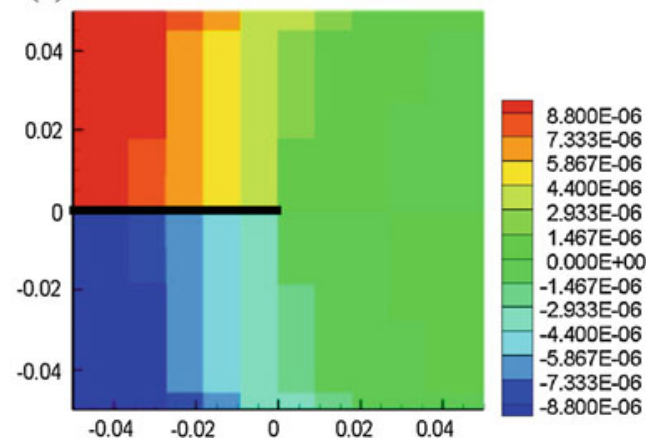

Fig. 8 Comparison of vertical displacements obtained with: a the FEM (about 30,000 nodes), b peridynamics using $\delta=$ $1.5 \mathrm{~mm}, m=9$ (about 360,000 nodes), and c peridynamics using $\delta=24 \mathrm{~mm}, m=3$ (about 144 nodes) (geometry and loading as given in Fig. 6). The legend is in meters (a)

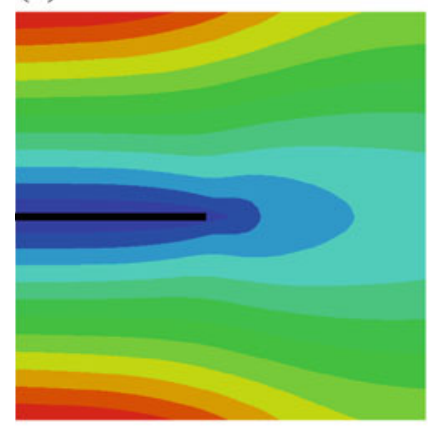

(b)

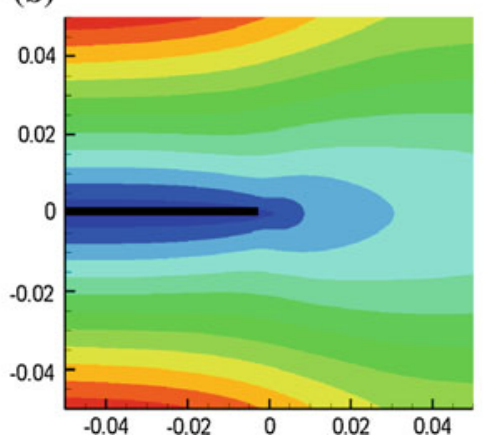

(c)

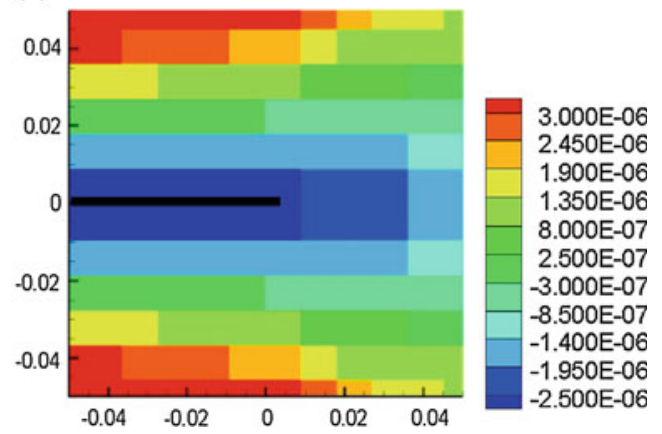

Fig. 9 Comparison for horizontal displacements obtained with: a the FEM, b peridynamics using $\delta=1.5 \mathrm{~mm}, m=9$, and $\mathbf{c}$ peridynamics using $\delta=24 \mathrm{~mm}, m=3$ (geometry and loading as given in Fig. 6). The legend is in meters

of the classical model. Thus, when the intention is to obtain peridynamic J-integral values close to the classical ones, an $m$ of about 6 and a horizon size smaller than $6 \mathrm{~mm}$ (a ratio of at least $1 / 10$ to the crack length, and $1 / 20$ to the sample dimensions) are good choices.

Remark the peridynamic "skin effect" mentioned in Sect. 2 exists on the crack surfaces. Hence, we expect peridynamic J-integral results to be higher than the FEM results since we effectively have a softer material (Young's modulus value half of that in the bulk) around the crack tip than the material in the bulk. We assigned a softer material to a thin region, of thickness equal to $1.5 \mathrm{~mm}$, the same as the size of the smallest peridynamic horizon used here, around the crack line in the FEM model and the J-integral value from the Abaqus calculation increased by about $2 \%$.

\subsection{Double-notched specimen}

In this section, we consider a double-notch plate with dimensions $20 \mathrm{~cm} \times 10 \mathrm{~cm}$. The length of each notch is $5 \mathrm{~cm}$. By making use of symmetry, we can reduce the problem to analyzing the same configuration as before (see Fig. 6) except that now we impose symmetry conditions on the displacements along the righthand boundary (see Fig. 11). We use the same integral contour as in Sect. 5.1 and three different horizon sizes $\delta=6 \mathrm{~mm}, \delta=3 \mathrm{~mm}$, and $\delta=1.5 \mathrm{~mm}$ and $m=3,6$, and 9 to observe how convergence is influenced by the presence of the symmetry boundary condition. While in a classical model discretized with finite elements the symmetry boundary condition is simply imposed on the boundary nodes where zero horizontal displacements are enforced, in a nonlocal peridynamic model this type of condition is more delicate. In principle, in 
Table 2 Peridynamic J-integral values for the single edge notched specimen

\begin{tabular}{llllll}
\hline & $\delta=24 \mathrm{~mm}$ & $\delta=12 \mathrm{~mm}$ & $\delta=6 \mathrm{~mm}$ & $\delta=3 \mathrm{~mm}$ & $\delta=1.5 \mathrm{~mm}$ \\
\hline$m=3$ & $30.15 \mathrm{Pam}$ & $23.64 \mathrm{Pam}$ & $21.74 \mathrm{Pam}$ & $20.83 \mathrm{Pam}$ & $20.41 \mathrm{Pam}$ \\
$m=6$ & $26.52 \mathrm{Pam}$ & $23.05 \mathrm{Pam}$ & $21.54 \mathrm{Pam}$ & $20.85 \mathrm{Pam}$ & $20.51 \mathrm{Pam}$ \\
$m=9$ & $26.17 \mathrm{Pam}$ & $22.95 \mathrm{Pam}$ & $21.53 \mathrm{Pam}$ & $20.86 \mathrm{Pam}$ & $20.53 \mathrm{Pam}$ \\
\hline
\end{tabular}

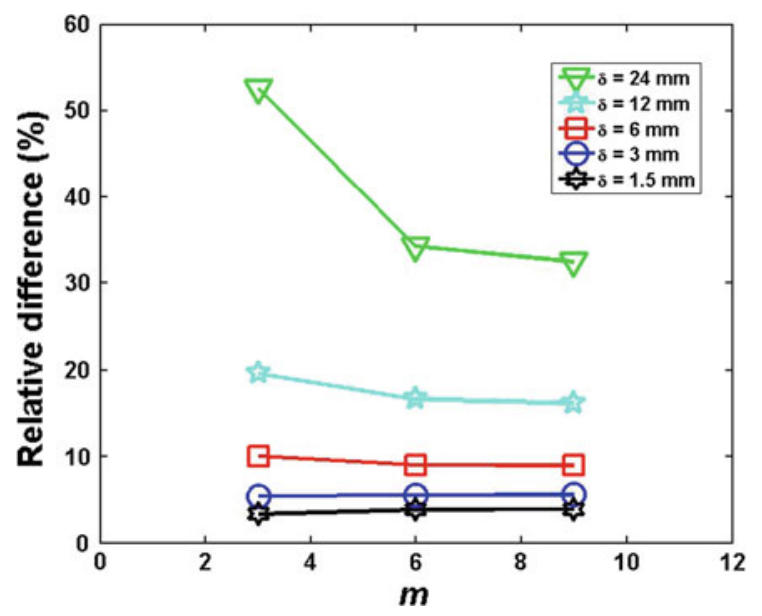

Fig. 10 The relative difference between the peridynamic results for the single-notch sample and the classical J-integral obtained with the FEM (Abaqus)

the discrete peridynamic model we should enforce the boundary nodes to have zero horizontal displacements, but in addition to that, for a sliver of thickness $\delta$ on the corresponding boundary we should also prescribe the horizontal displacement field (see Silling 2000 for a discussion on nonlocal Dirichlet boundary conditions). The problem is that those values are not known. Ways around this are possible: for example, one could set zero horizontal displacements for all nodes in the region of thickness $\delta$, and in the limit of the horizon going to zero this willapproach the actual symmetry condition desired; alternatively, one could impose zero horizontal displacements only on the boundary nodes, just like in a finite element model. We choose the latter strategy because of simplicity and similarity to the way boundary conditions are imposed for the classical model.

The finite element solution from Abaqus with special crack-tip elements is $12.90 \mathrm{~Pa}$.

As shown in Fig. 12, the strain energy density obtained with peridynamics for a sufficiently small horizon size is very close to that given by a converged FEM solution. However, some high strain energy density values are observed from the peridynamic result on

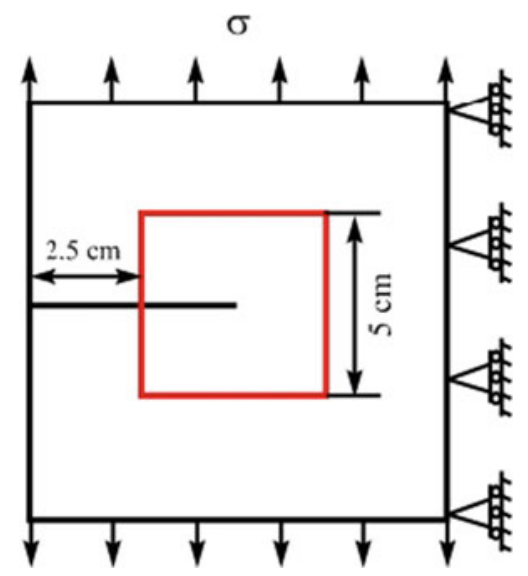

Fig. 11 The double edge notched specimen with symmetric boundary conditions and the J-integral contour

the right-hand boundary, where the symmetric boundary condition is applied (see Fig. 12). This influences, to a certain extent, the results for the J-integral, as we will see below. We also notice that the difference in brightness between the FEM and peridynamics results are not due to differences in the values, but are caused by differences in the colors used by Abaqus (for the FEM results) and Tecplot (which we used to post-process the peridynamic results).

The $m$-convergence trends are the same for all three cases as shown in Fig. 13. We stress again that in $m$-convergence studies, the peridynamic approximate solutions are not supposed to converge to the classical solution, but to the nonlocal solution for the particular horizon size for which the $m$-convergence study is performed. Since we do not have the exact solution for the nonlocal problem, we compare the results with the classical solution for illustration purposes. See also the comments in Bobaru and Duangpanya (2010, 2012), on a peridynamic model for diffusion processes. The data in Table 3 shows that the peridynamic solutions are all larger than the FEM result, which is consistent with the results obtained in section 5.1. The effectively softer material around the crack tip (induced by the peridy- 
(a)

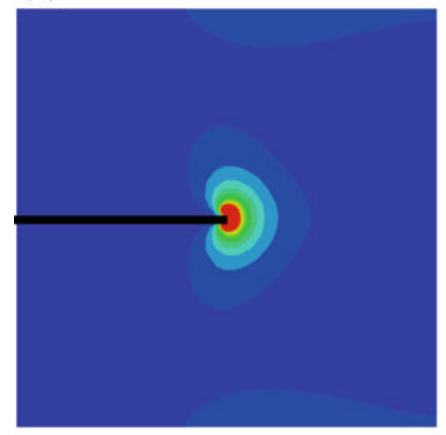

(b)

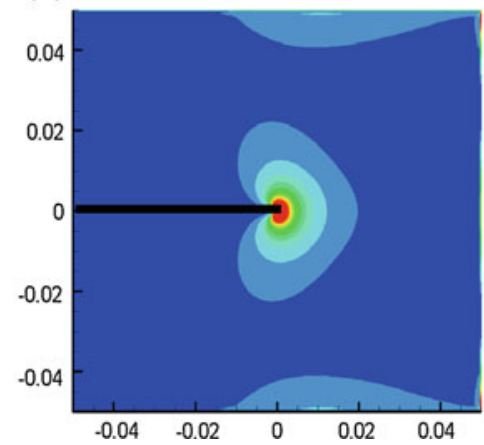

(c)

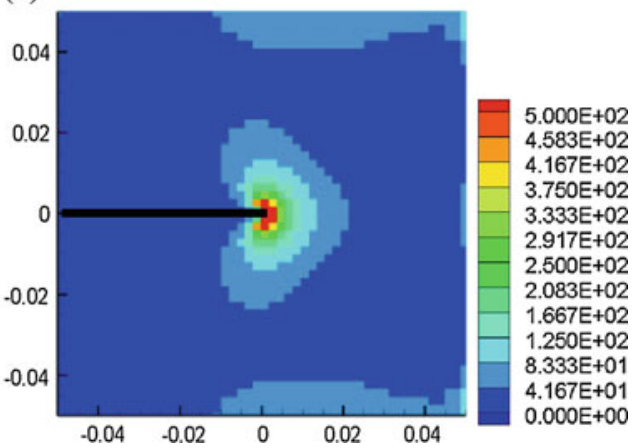

Fig. 12 Strain energy density results with: a FEM (30,000 nodes), b peridynamics with $\delta=1.5$ mm, $m=9$ (about 360,000 nodes) c peridynamics with $\delta=6 \mathrm{~mm}, m=3$ (about 2700 nodes)

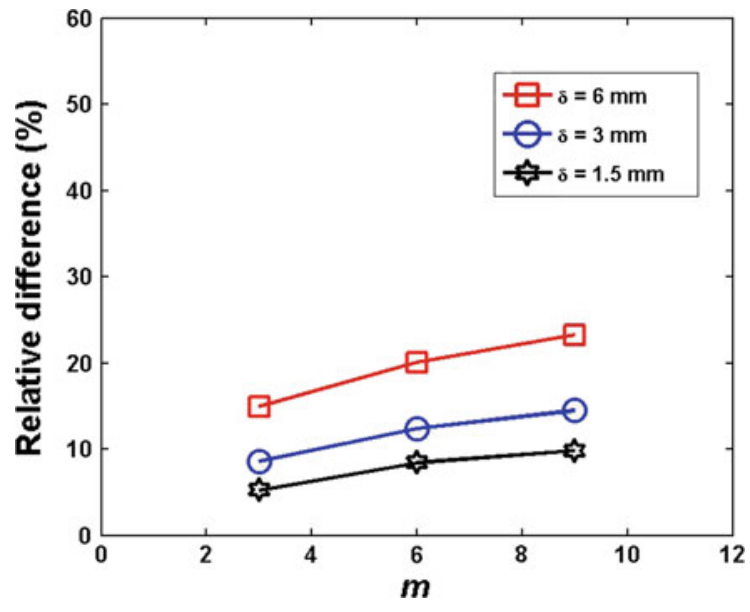

Fig. 13 The relative difference between peridynamic results for the double edge-notch (with symmetric boundary conditions) and the classical J-integral value obtained from a FEM (Abaqus) analysis

namic "skin effect") is responsible for this. In addition, for a given $m$-value, the smaller the horizon size the closer the peridynamic result is to the classical value, confirming that $\delta$-convergence takes place. With a sufficiently small horizon, the relative difference between the classical J-integral value and the peridynamic Jintegral becomes smaller than a few percentages. The differences between the peridynamic results and the classical J-integral value are, however, larger now than before, for a similar horizon size. The likely motive for this change is the imposition of the symmetric boundary conditions. The influence of the imposition of the symmetric boundary conditions on the calculation of the peridynamic $\mathrm{J}$-integral is further investigated in the next
Table 3 Convergence study with double edge notched specimen

\begin{tabular}{llll}
\hline & $\delta=6 \mathrm{~mm}$ & $\delta=3 \mathrm{~mm}$ & $\delta=1.5 \mathrm{~mm}$ \\
\hline$m=3$ & $14.83 \mathrm{Pam}$ & $14.01 \mathrm{Pam}$ & $13.58 \mathrm{Pam}$ \\
$m=6$ & $15.49 \mathrm{Pam}$ & $14.50 \mathrm{Pam}$ & $13.99 \mathrm{Pam}$ \\
$m=9$ & $15.90 \mathrm{Pam}$ & $14.77 \mathrm{Pam}$ & $14.17 \mathrm{Pam}$ \\
\hline
\end{tabular}

section by choosing different integration paths around the crack tip.

\subsection{The path-independence of the peridynamic J-integral}

As the peridynamic J-integral over a closed contour in a micro-elastic material is zero, analytically, the peridynamic J-integral is path independent. We test here different contours to observe if, and by how much, the value of the peridynamic J-integral (computed using the algorithm in Table 1) changes with changing the integration contour. For these tests we use the smallest horizon and finest grid from the computations above $(m=$ 9 and $\delta=1.5 \mathrm{~mm}$ ) and perform calculations for the single edge-notch problem and the double edge-notch problem with symmetry boundary conditions. We use the three different contours shown in Fig. 14: the closest contour to the crack tip that remains feasible from the point of view of evaluating the integrals in Eq. (20) (see also Fig. 4), another away from the crack tip and the boundaries, and finally one near the boundaries, but at least one horizon size away from the boundaries to remain feasible. Thus, contour (a) in Fig. 14 is one horizon size away from the crack tip, contour (b) is in the middle of the structure, and contour (c) is one horizon away from the boundary of the sample (Table 4). 
Fig. 14 Three different integral contours to compute peridynamic $\mathrm{J}$ integral (top). Feasible and unfeasible contours (bottom row) for the computation of the nonlocal J-integral (see the remark at the end of Sect. 3) near the crack tip and near a domain boundary
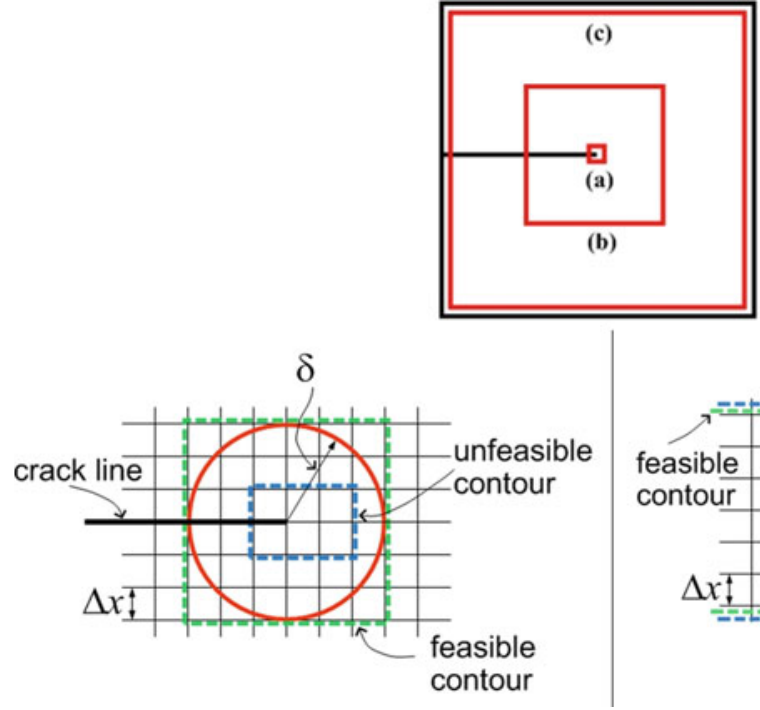

Table 4 Values of the peridynamic J-integral on the three different contours from Fig. 14

\begin{tabular}{llll}
\hline & Contour (a) & Contour (b) & Contour (c) \\
\hline Single edge-notch & 20.11 Pam & 20.54 Pam & 19.84 Pam \\
Double & $13.88 \mathrm{Pam}$ & $14.17 \mathrm{Pam}$ & $13.37 \mathrm{Pam}$ \\
$\quad \begin{array}{l}\text { edge-notch } \\
\text { (using }\end{array}$ & & & \\
$\quad \begin{array}{l}\text { symmetric } \\
\text { b.c.'s) }\end{array}$ & & & \\
\hline
\end{tabular}

We measure the relative difference between the values obtained based on contour (b) and those on contours (a) and (c). As shown in Table 5, the relative differences between contour (a) and contour (b) for both the single edge-notch and double edge-notch specimens are very similar, equal to about $2 \%$. However, the relative difference between contour (c) and contour (b) is larger in the double-edge case (with symmetric boundary conditions) than in the single edge-notch case. The reason is likely the way the nonlocal Dirichlet boundary condition is enforced and the peridynamic "skin-effect". Notice that the results on contour (c) are actually closer to the classical results obtained with the FEM, but these trends should not be misinterpreted. The horizon size, the number of nodes in the horizon, and the type of boundary conditions, all "influence" the approximate solutions of nonlocal models, and these results may be lower than a classical value for some $m$-values, and higher for some other $m$-values. We recall that in $m$-convergence, as $m$ tends to infinity, the numerical
Table 5 Relative difference between the peridynamic J-integral on contours (a) and (c) in Fig. 14 and the corresponding values obtained on contour (b)

\begin{tabular}{lll}
\hline & Contour (a) & Contour (c) \\
\hline Single edge-notch & $2.09 \%$ & $3.41 \%$ \\
$\begin{array}{l}\text { Double edge-notch } \\
\text { (using symmetric }\end{array}$ & $2.05 \%$ & $5.65 \%$ \\
boundary conditions) & & \\
\hline
\end{tabular}

approximation is supposed to approach the exact solution of the nonlocal problem and not the exact solution of the classical problem. It is known that, in certain cases, nonlocal results may be closer to the classical results for some $\delta_{1}$ and $m_{1}$, and higher for a $\delta_{2}<\delta_{1}$ and $m_{2}>m 1$. This means that we may match the exact classical solution for some finite $\delta_{3}$ in $\left[\delta_{2}, \delta_{1}\right]$ and $m_{3}$ in $\left[m_{1}, m_{2}\right]$ (see also Bobaru and Duangpanya 2010, 2012).

In conclusion, a contour for computing the peridynamic J-integral needs to avoid the boundary region because of the skin effect and imposition of nonlocal Dirichlet boundary conditions. On the other hand, the contour may be selected near the crack tip, with the only condition being the feasibility requirement mentioned in the remark in Sect. 3 and shown in Fig. 14.

\section{Conclusions}

In this paper we derived the 2D peridynamic J-integral formulation based on the infinitesimal virtual extension 
of the mode-I crack. We discussed the computation of the peridynamic J-integral value which involves a double integral in contrast with the classical J-integral. We showed that the peridynamic J-integral converges to the classical J-integral formulation when the horizon size goes to zero. Using a simple discretization, we performed convergence studies ( $m$-convergence and $\delta$-convergence) for two specimens: a single edge-notch and a double edge-notch sample, for which symmetry boundary conditions were enforced. We observed that the peridynamic results approach within a few percentages the classical J-integral values obtained using the FEM with special element around the crack tip, when the horizon size is less than about $1 / 20$ of the crack size and sample size. In particular, we discussed the influence of the peridynamic "skin-effect" around the crack tip and along the boundaries, on the value of the peridynamic J-integral. We computed the peridynamic Jintegral along different paths and very small variations were seen (attributable to the approximation error) between the contours, except when the integration path was within a horizon distance from the boundaries. Due to nonlocality, special care also needs to be paid when symmetric boundary conditions are imposed.

Acknowledgments The WH, YDH, and FB are thankful for the financial support offered through research contracts between UNL and the ARO (Dr. Larry Russell), ARL (project coordinators Dr. C.F. Yen and Dr. C. Randow), ARO award number 58450EG, and Sandia National Laboratories (contract number 568428). Sandia National Laboratories is a multi-program laboratory operated by Sandia Corporation, a wholly owned subsidiary of Lockheed Martin company, for the U.S. Department of Energy's National Nuclear Security Administration under contract DE-AC04-94AL85000. The computations in this work were completed utilizing the Holland Computing Center of the University of Nebraska.

\section{References}

Bobaru F (2007) Influence of van der Waals forces on increasing the strength and toughness in dynamic fracture of nanofiber networks: a peridynamic approach. Model Simul Mater Sci Eng 15:397-417

Bobaru F, Duangpanya M (2010) The peridynamic formulation for transient heat conduction. Int $\mathrm{J}$ Heat Mass Transfer 53:4047-4059

Bobaru F, Duangpanya M (2012) A peridynamic formulation for transient heat conduction in bodies with evolving discontinuities. J Comput Phys 231(7):2764-2785

Bobaru F, Ha YD (2011) Adaptive refinement and multiscale modeling in $2 \mathrm{D}$ peridynamics. Int $\mathrm{J}$ Mult Comput Eng 9(6):635-660
Bobaru F, Hu W (2012) The meaning, selection, and use of the peridynamic horizon and its relation to crack branching in brittle materials. Int J Fract. doi:10.1007/ s10704-012-9725-z

Bobaru F, Yang M, Alves LF, Silling SA, Askari E, Xu J (2009) Convergence, adaptive refinement, and scaling in 1D peridynamics. Int J Numer Meth Eng 77(6):852-877

Foster JT, Silling SA, Chen WW (2010) Viscoplasticity using peridynamics. Int J Numer Meth Eng 81:1242-1258

Foster J, Silling SA, Chen WW (2011) An energy based failure criterion for use with peridynamic states. Int J Mult Comp Eng 9:675-688

Ha YD, Bobaru F (2010) Studies of dynamic crack propagation and crack branching with peridynamics. Int J Fract 162:229-244

Ha YD, Bobaru F (2011) Characteristics of dynamic brittle fracture captured with peridynamics. Eng Fract Mech 78:11561168

Hu W, Ha YD, Bobaru F (2012) Peridynamic simulations of dynamic fracture in unidirectional fiber-reinforced composites. Comput Methods Appl Mech Eng. 217-220: 247-261

Hu W, Ha YD, Bobaru F (2011) Modeling dynamic fracture and damage in fiber-reinforced composites with peridynamics. Int J Mult Comput Eng 9:707-726

Hutchinson JW (1968) Singular behavior at the end of a tensile crack tip in a hardening material. J Mech Phys Solids 16:13-31

Kilic B (2008) Peridynamic theory for progressive failure prediction in homogeneous and heterogeneous materials. Ph.D. thesis, Department of Aerospace and Mechanical Engineering, University of Arizona, pp 68

Kilic B, Agwai A, Madenci E (2009) Peridynamics theory for progressive damage prediction in center-cracked composite laminates. Compos Struct 90:141-151

Lehoucq RB, Silling SA (2008) Force flux and the peridynamic stress tensor. J Mech Phys Solids 56:1566-1577

Macek RW, Silling SA (2007) Peridynamics via finite element analysis. Finite Elem Eng Des 43:1169-1178

Rice JR (1968) A path independent integral and the approximate analysis of strain concentration by notches and cracks. J Appl Mech 9:379-386

Rice JR, Rosengren GF (1968) Plane strain deformation near a crack tip in a power-law hardening material. J Mech Phys Solids $16: 1-12$

Silling SA (2000) Reformulation of elasticity theory for discontinuities and long-range forces. J Mech Phys Solids 48:175-209

Silling SA, Askari E (2005) A meshfree method based on peridynamic model of solid mechanics. Comput Struct 83:1526-1535

Silling SA, Bobaru F (2005) Peridynamic modeling of membranes and fibers. Int J Nonlinear Mech 40:395-409

Silling SA, Lehoucq RB (2010) Peridynamic theory of solid mechanics. Adv Appl Mech 44:73-168

Xu J, Askari E, Weckner O, Silling SA (2008) Peridynamic analysis of impact damage in composite laminates. J Aerosp Eng 21:187-194 